\title{
Utility of Indocyanine Green Video Angiography for Sylvian Fissure Dissection in Subarachnoid Hemorrhage Patients - Sylvian ICG Technique
}

\author{
Hiroyuki ToI, ${ }^{1}$ Nobuhisa MATSUSHITA,,${ }^{1}$ Yukari OGAwA, ${ }^{1}$ Keita KINOSHITA, ${ }^{1}$ \\ Kohei SATOH, ${ }^{1}$ Hiroki TAKaI, ${ }^{1}$ Satoshi HIRAI, ${ }^{1}$ Keijiro HARA, ${ }^{1}$ \\ Shunji MATSUBARA, ${ }^{1}$ and Masaaki $\mathrm{UNO}^{1}$
}

${ }^{1}$ Department of Neurosurgery, Kawasaki Medical School, Kurashiki, Okayama, Japan

\begin{abstract}
Indocyanine green (ICG) emits fluorescence in the far-red domain under light excitation. ICG video angiography (ICG-VA) has been established as a useful method to evaluate blood flow in the operative field. We report the usefulness of ICG-VA for Sylvian fissure dissection in patients with subarachnoid hemorrhage (SAH). Subjects comprised 7 patients who underwent ICG-VA before opening the Sylvian fissure during neck clipping for ruptured cerebral aneurysm. We observed contrasted Sylvian veins before opening the Sylvian fissure using surgical microscopes. This procedure was termed "Sylvian ICG". We observed ICG fluorescence quickly in all cases. Sylvian veins that appeared unclear in the standard microscopic operative field covered with subarachnoid hemorrhage were extremely clearly depicted. These Sylvian ICG findings were helpful in identifying entry points and the dissecting course of the Sylvian fissure. At the time of clipping, no residual fluorescence from Sylvian ICG was present, and aneurysm clipping was not impeded. Sylvian ICG for SAH patients is a novel technique to facilitate dissection of the Sylvian fissure. We believe that this technique will contribute to improved safety of clipping surgery for ruptured aneurysms.
\end{abstract}

Key words: indocyanine green video angiography, subarachnoid hemorrhage, Sylvian fissure, Sylvian veins

\section{Introduction}

Indocyanine green (ICG) emits fluorescence in the far-red domain under light excitation. ICG video angiography (VA) has been established as a useful method to evaluate blood flow in the operative field, ${ }^{1,2)}$ and is commonly used to observe arteries in cerebrovascular surgeries such as aneurysm clipping, ${ }^{2-5)}$ removal of arteriovenous malformation, ${ }^{6,7)}$ carotid endarterectomy, ${ }^{4,8)}$ and bypass surgery. ${ }^{9,10)}$

In the present study, we performed ICG-VA prior to clipping in patients with aneurysmal subarachnoid hemorrhage (SAH) before dissecting the Sylvian fissure, to evaluate the Sylvian veins and decide on the optimal course of dissection to the Sylvian fissure. We report on the usefulness of ICG-VA for Sylvian fissure dissection.

Received July 21, 2017; Accepted September 22, 2017

Copyright@ 2018 by The Japan Neurosurgical Society This work is licensed under a Creative Commons AttributionNonCommercial-NoDerivatives International License.

\section{Materials and Methods}

Subjects selected for analysis were seven patients for whom ICG-VA was performed before opening the Sylvian fissure in neck clipping for ruptured cerebral aneurysm in our department between May 2015 and May 2016. The demographic and clinical characteristics of these patients are shown in Table 1. We excluded cases who underwent coiling and unruptured aneurysms. All patients underwent computed tomography (CT), magnetic resonance imaging (MRI), and cerebral angiography, then $\mathrm{SAH}$ was diagnosed and the ruptured aneurysm was detected. We decided to perform clipping considering the condition of the individual patient. All patients underwent surgery in the acute period, with the exception of one case of delayed surgery. The research protocol of the study was approved by the ethics committee of Kawasaki Medical School, Kurashiki, Okayama, Japan. All patients gave informed consent.

\section{Sylvian ICG procedure}

All patients underwent frontotemporal craniotomy and the dura was opened in the usual manner. 
Table 1 Demographic and clinical characteristics of the seven patients with Sylvian ICG

\begin{tabular}{|c|c|c|c|c|c|c|c|c|}
\hline $\begin{array}{c}\text { Case } \\
\text { no. }\end{array}$ & $\begin{array}{c}\text { Age } \\
\text { (years) }\end{array}$ & Sex & $\begin{array}{c}\text { Location of } \\
\text { aneurysm }\end{array}$ & $\mathrm{H}-\mathrm{H}$ grade & Fisher group & Onset to op (hours) & $\begin{array}{c}\text { mRS score } \\
\text { at discharge }\end{array}$ & $\begin{array}{l}\text { Length of hospital } \\
\text { stay (days) }\end{array}$ \\
\hline 1 & 69 & $\mathrm{~F}$ & PCOM & 2 & 3 & 5 & 1 & 26 \\
\hline 2 & 52 & $\mathrm{~F}$ & MCA & 3 & 3 & 9 & 4 & 32 \\
\hline 3 & 51 & $\mathrm{~F}$ & AChA & 2 & 2 & 17 & 1 & 39 \\
\hline 4 & 68 & M & MCA & 1 & 1 & Delayed op* & 1 & 18 \\
\hline 5 & 66 & $\mathrm{~F}$ & PCOM & 3 & 4 & 17 & 1 & 28 \\
\hline 6 & 70 & $\mathrm{M}$ & $\mathrm{AChA}$ & 2 & 2 & 63 & 0 & 26 \\
\hline 7 & 51 & $\mathrm{~F}$ & AChA & 2 & 3 & 32 & 0 & 15 \\
\hline
\end{tabular}

*: operated at 13 days after SAH onset, PCOM: posterior communicating artery aneurysm, MCA: middle cerebral artery aneurysm, AchA: anterior choroidal artery aneurysm, H-H grade: Hunt and Hess Grade, mRS: modified Rankin Scale.

A solution of ICG of $25 \mathrm{mg}$ in $10 \mathrm{~mL}$ of distilled water was prepared. After intravenous injection of $3 \mathrm{~mL}$ of ICG solution (including $7.5 \mathrm{mg}$ of ICG) and $10 \mathrm{~mL}$ of saline flush, the brain surface and contrastenhanced Sylvian veins were observed before opening the Sylvian fissure using surgical microscopes OPMI PENTERO 900 (Carl Zeiss Meditec, Berlin, Germany). We named this procedure "Sylvian ICG". We next started to open the Sylvian fissure with reference to the findings from Sylvian ICG, and performed neck clipping of the ruptured cerebral aneurysm in the usual manner. Intraoperatively, we also performed ICG-VA just before and after clipping to confirm complete occlusion of the aneurysms.

Informed consent for the procedure was obtained from the families of all patients. This method was approved by the ethics screening committee of our institution.

\section{Results}

Mean age of the $7 \mathrm{SAH}$ patients was 61.0 years (range, 51-70 years), and there were 5 female and 2 male patients. Location of the ruptured aneurysm was as follows: posterior communicating arteries, 2 cases; anterior choroidal arteries, 3 cases; and middle cerebral arteries, 2 cases. No anterior communicating artery or posterior circulation aneurysms were encountered. Hunt and Hess grade ${ }^{11)}$ was as follows: grade 1, 1 patient; grade 2, 4 patients; and grade 3,2 patients. No patients showed grade 4 or 5 aneurysms. Fisher group ${ }^{12)}$ was as follows: group 1, 1 patient; group 2, 2 patients; group 3, 3 patients; and group 4, 1 patient. Mean period from $\mathrm{SAH}$ onset to surgery was $22.2 \mathrm{~h}$ (range, 5-63 h), after excluding an outlier case with delayed surgery (Case 4) performed on day 13 after onset. No surgery-related mortality or rebleeding of aneurysms were encountered. Mean duration of hospitalization was 26.3 days (range, 15-39 days). At discharge,
6 patients $(85.7 \%)$ showed good recovery (modified Rankin Scale score, 0-2). ${ }^{13)}$

Concerning intraoperative findings from Sylvian ICG, we successfully observed rapid ICG fluorescence in all cases. Sylvian veins that were unclear under the usual microscopic operative field covered with SAH were detected extremely clearly. Those Sylvian ICG findings were helpful in selecting entry points and the course to dissection of the Sylvian fissure. By understanding the venous patterns, we were able to perform the surgery without any injuries to the Sylvian veins. After exposing the aneurysm, we also performed ICG-VA just before aneurysm clipping. No residual fluorescence from the preceding Sylvian ICG remained by that time, and no influence on aneurysm clipping was seen. We confirmed complete clipping in all cases. No patients experienced any complications from the Sylvian ICG procedure.

\section{Illustrative Cases}

\section{Case 2}

A 52-year-old woman presented to the emergency room complaining of acute onset of severe headache. On admission, level of consciousness was E3V2M6 on the Glasgow Coma scale. Initial head CT revealed diffuse SAH and angiography demonstrated left middle cerebral artery aneurysm. We diagnosed SAH (Hunt and Hess grade 3, Fisher group 4), and decided on treatment with clipping. After frontotemporal craniotomy and opening of the dura mater (Fig. 1A), Sylvian ICG was performed before arachnoid incision. This case demonstrated clear Sylvian ICG (Fig. 1B), allowing safe dissection of the Sylvian fissure (Fig. 1C). The venous phase of preoperative cerebral angiography and intraoperative Sylvian ICG are compared in Fig. 2. Sylvian ICG accurately represented simple Sylvian veins. Clipping was completed, and no additional 
neurological symptoms appeared postoperatively. However, symptomatic vasospasm and paralysis of the left lower extremity developed, the patient returned to everyday life after rehabilitation therapy.

\section{Case 5}

A 66-year-old woman presented with acute onset of headache and disturbance of consciousness. On admission, level of consciousness was E3V4M6 on the Glasgow Coma Scale. Initial head CT revealed diffuse $\mathrm{SAH}$ and angiography demonstrated aneurysm of the
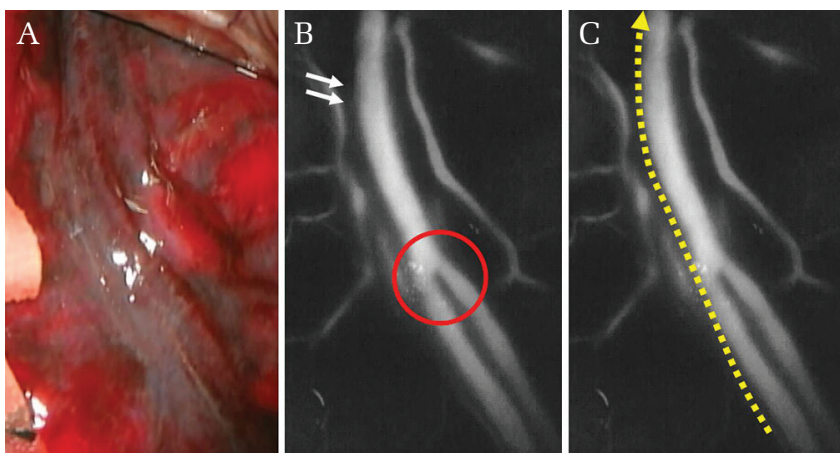

Fig. 1 Case 2. Illustrative case of a patient with ruptured left middle cerebral artery aneurysm. (A) Intraoperative microscopic findings of the Sylvian fissure before Sylvian ICG. Sylvian veins are unclear because of diffuse subarachnoid. (B) Intraoperative view from the same position after Sylvian ICG, demonstrating Sylvian veins extremely clearly. The double arrow shows safety space between the superficial Sylvian veins for dissection, confirming the true Sylvian fissure. The red circle shows tortuosity and junction of the veins. (C) The yellow line represents the course of dissection selected.
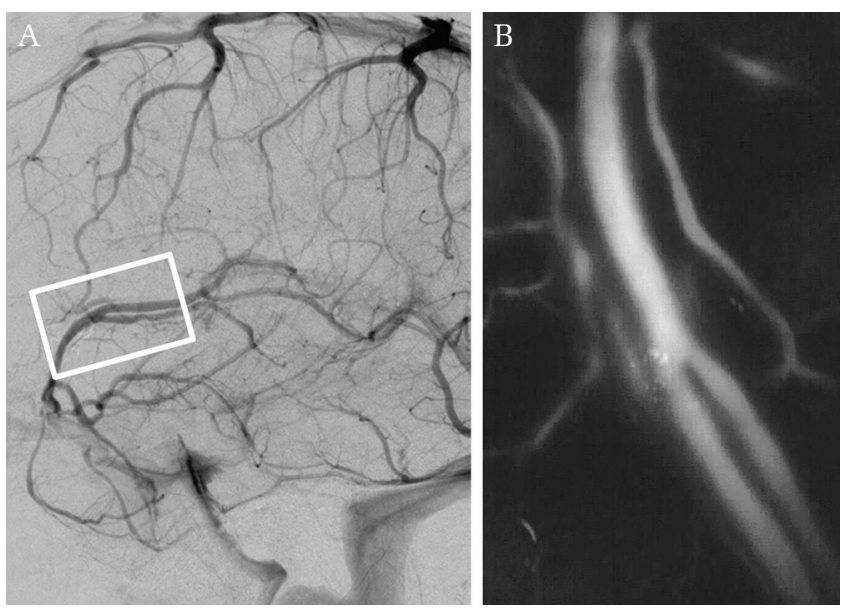

Fig. 2 Case 2. The venous phase of preoperative cerebral angiography (A) and intraoperative Sylvian ICG (B) are compared. Sylvian ICG accurately represent simple Sylvian veins. The white square in Fig. 2A shows the same part of the Sylvian vein observed by Sylvian ICG. right posterior communicating artery. The patient was diagnosed with SAH (Hunt and Hess grade 3, Fisher group 4). The decision was made to perform clipping in order to prevent aneurysmal rerupture. After frontotemporal craniotomy and opening of the dura mater (Fig. 3A), we performed Sylvian ICG before arachnoid incision. Although thick SAH was present around the veins, fluorescence of the Sylvian veins was clearly observed following fluorescence of the cortical arteries. Intraoperative findings before and after Sylvian ICG in this case are shown in Figs. $3 \mathrm{~A}$ and $3 \mathrm{~B}$. We started to open the Sylvian fissure with reference to Sylvian ICG findings (Fig. 3C), then complete clipping was performed. The venous phase of preoperative cerebral angiography and intraoperative Sylvian ICG are compared in Fig. 4. Sylvian ICG accurately represented complex Sylvian veins. The postoperative course was uneventful and she returned to a social life equivalent to her preoperative level.

\section{Discussion}

Raabe et al. first described ICG-VA for neurosurgery in $2003,{ }^{1)}$ using the method to observe the cerebral arteries. Since 2005, many reports have suggested the usefulness of ICG-VA for aneurism surgery. ${ }^{2-5)}$ This approach allows clear visualization of intraaneurysmal blood flow. Based on such findings, ICG-VA has come into wide use for improving the efficacy and safety of various neurosurgical procedures.
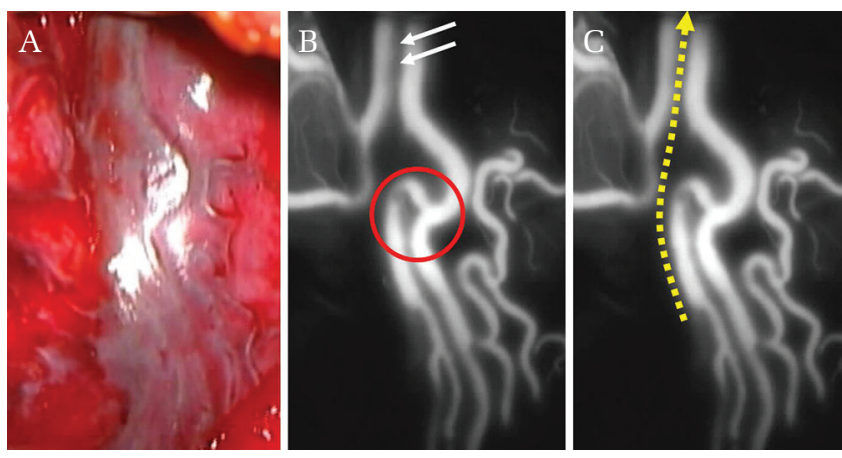

Fig. 3 Case 5. Illustrative case of a patient with ruptured right posterior communicating artery aneurysm. (A) Intraoperative microscopic findings of the Sylvian fissure before Sylvian ICG. Sylvian veins are unclear because of diffuse subarachnoid hemorrhage. (B) Intraoperative view from the same position after Sylvian ICG demonstrates the Sylvian veins extremely clearly. The double arrow shows safety space between the superficial Sylvian veins for dissection. The red circle shows tortuosity and junction of the veins. Reflection of the microscopic light has no influence. (C) The yellow line represents the course of dissection selected. 
In bypass surgery, ICG-VA provides reliable, rapid intraoperative assessment of bypass patency, contributing to a reduced incidence of early bypass graft failure. ${ }^{9,10}$ The procedure is also used in carotid endarterectomy $\mathrm{y}^{4,8)}$ and surgeries for cerebral arteriovenous malformations, ${ }^{6,7,14)}$ spinal vascular malformations, ${ }^{12)}$ and brain tumors. ${ }^{15-17)}$

However, few reports have examined the relationship between ICG-VA and veins. The present study applied ICG-VA to the cerebral venous system, using this "Sylvian ICG" technique to observe Sylvian veins covered with SAH. Motoyama et al. reported a similar case involving a patient with thin SAH. ${ }^{18)}$ We believe that our series offers the first report of ICG for Sylvian veins covered with SAH. Seven aneurysmal SAH patients underwent clipping with this procedure, and Sylvian ICG images were clearly observed in all cases. The visibility of ICG fluorescence in the venous system was extremely good, similar to that in arterial system. Sylvian ICG facilitates clear identification of the Sylvian veins, whereas detection of veins surrounding thick SAH is difficult in the usual microscopic surgical field because of the similarity in coloration to hematoma. The primary objective of Sylvian ICG is to accurately ascertain the position of the superficial vein that is difficult to see with hematoma before arachnoid incision. After the superficial arachnoid incision, at the stage of opening the Sylvian fissure, it is easy to detect the position of the veins, because hematoma is gradually washed away. For this reason, although it is possible to repeat Sylvian ICG at the deep part of the Sylvian fissure, requirement of performing it

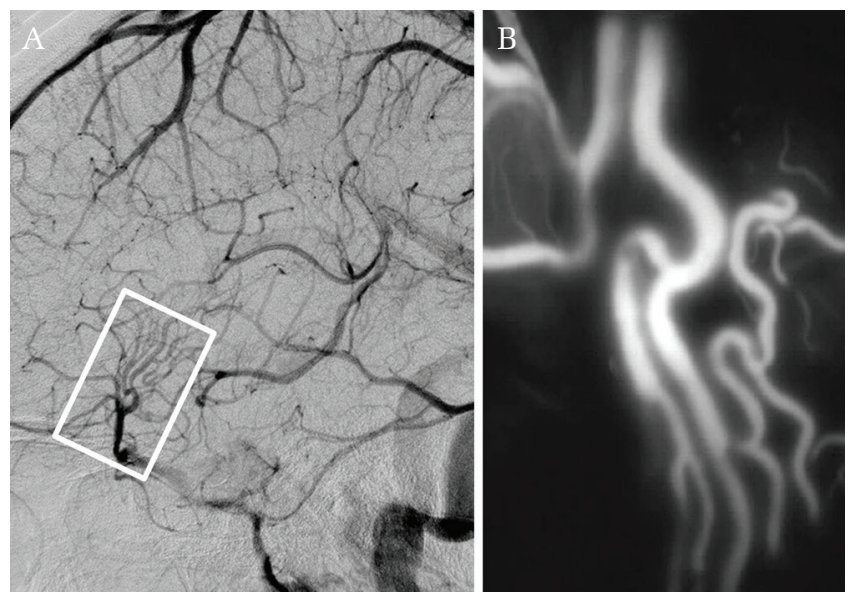

Fig. 4 Case 5. The venous phase of preoperative cerebral angiography (A) and intraoperative Sylvian ICG (B) are compared. Sylvian ICG accurately represent complex Sylvian veins. The white square in Fig. 4A shows the same part of the Sylvian vein observed by Sylvian ICG. is relatively low. The schemas of intraoperative findings before and after Sylvian ICG are shown in Fig. 5.

Sylvian ICG offers three benefits. First, the crevices of the Sylvian veins can be precisely detected as a black part of the Sylvian fissure. We can thus select the best entry points and course of dissection to the Sylvian fissure. Second, Sylvian ICG demonstrates tortuosity and junction of the veins, allowing avoidance of points that may be disadvantageous to dissect. In our experience, there were no cases in which venous junctions were unclear
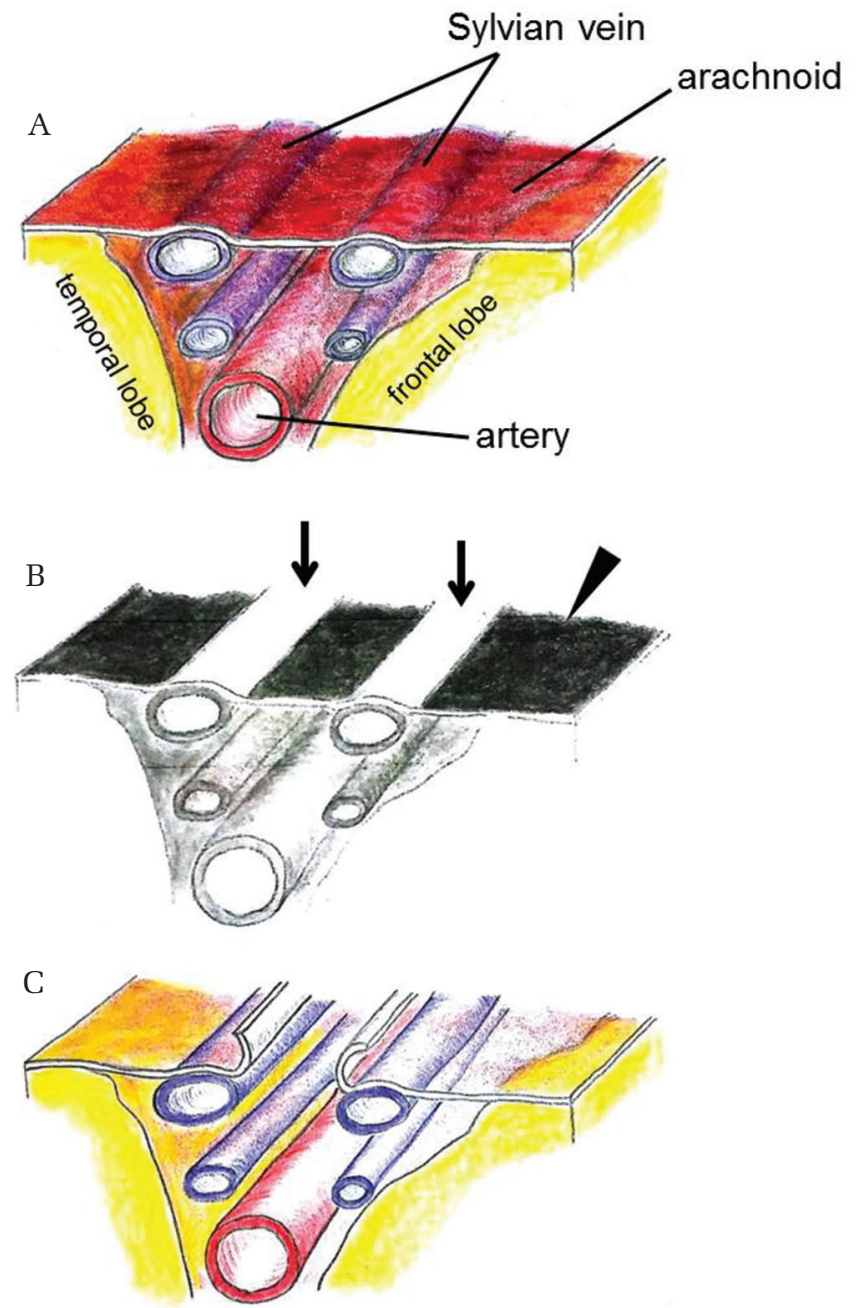

Fig. 5 The schemas of intraoperative findings before and after Sylvian ICG. Before the procedure, it is difficult to identify Sylvian veins because they are of the same color as the surrounding hematoma (A). After Sylvian ICG, it is easy to identify superficial Sylvian veins (B). Superficial Sylvian veins are shown in white (arrows) and surrounding arachnoid with hematoma is shown in black (arrow head). After arachnoid incision, it is easy to identify deep Sylvian veins due to hematoma is washed away (C). 
to detect due to the strong flow. Also, because ICG is sensitive to "blood flow intensity" in venous systems, there is an advantage that ICG can visualize the difference in flow intensity in the veins of equivalent diameters. Third, venous patterns can be visualized in parts where reflection of the microscope light can interfere with visualization. These points are helpful for deciding on entry points and the course of dissection to the Sylvian fissure. Through understanding the venous vasculature, we are able to perform the surgery without injuring any Sylvian veins.

In addition to these advantages, none of our cases showed complications from this procedure. ICG is widely used in neurosurgical procedures, and is reasonably assumed to carry an extremely low risk. What is more, Sylvian ICG had no influence on aneurysm clipping. Although many operators use ICG-VA to confirm complete clipping, ICG used in Sylvian ICG does not remain in the arteries until that time, and so does not interrupt clipping.

In the ICG-VA, morphological assessments of arteries are likely to prove misleading if thick hematoma and/or brain tissue cover the target vessels. However, Sylvian ICG showed the veins clearly even in the presence of thick subarachnoid hemorrhage. This was attributed to venous vessel walls being thinner than those of arteries and existing closer to the brain surface.

It is thought that this technique is difficult to use for complex structural analysis of the deep part of Sylvian fissure. In the case of thin SAH, Sylvian ICG has little role and the impact is weak, however in the case of dense SAH, Sylvian ICG plays an extremely important role to accurately identify the superficial Sylvian veins before arachnoid incision.

Some limitations to the present study must be considered. The number of participants was still too small to allow detailed analysis. Further accumulation of data is needed to determine the optimal amount of ICG and timing of injection. Since various types of Sylvian veins exist, further studies are required to make this procedure more reliable.

\section{Conclusion}

Sylvian ICG for SAH patients is a novel technique to dissect the Sylvian fissure. We believe that this technique can contribute to improving the safety of clipping surgery for ruptured aneurysms.

\section{Conflicts of Interest Disclosure}

The authors declare no conflicts of interest associated with this manuscript. All authors have registered online Self-reported COI Disclosure Statement Forms through the website for JNS members.

\section{References}

1) Raabe A, Beck J, Gerlach R, Zimmermann M, Seifert V: Near-infrared indocyanine green video angiography: a new method for intraoperative assessment of vascular flow. Neurosurgery 52: 132-139; discussion 139, 2003

2) Raabe A, Nakaji P, Beck J, et al.: Prospective evaluation of surgical microscope-integrated intraoperative near-infrared indocyanine green videoangiography during aneurysm surgery. J Neurosurg 103: 982-989, 2005

3) Jing Z, Ou S, Ban Y, Tong Z, Wang Y: Intraoperative assessment of anterior circulation aneurysms using the indocyanine green video angiography technique. J Clin Neurosci 17: 26-28, 2010

4) Kamiyama K, Nakagawara J, Takada H, Fumoto K, Osato T, Nakamura H. Transformation of the vasa vasorum from normal vessel structures to atheroma nutrient vessels on ICG angiography of cervical carotid artery stenosis. Surgery for Cerebral Stroke 39: 413-419, 2011 (Japanese)

5) Takagi Y, Sawamura K, Hashimoto N. Intraoperative near-infrared indocyanine green videoangiography performed with a surgical microscope - applications in cerebrovascular surgery. European Neurological Review 3: 66-68, 2008

6) Khurana VG, Seow K, Duke D: Intuitiveness, quality and utility of intraoperative fluorescence videoangiography: Australian neurosurgical experience. Br J Neurosurg 24: 163-172, 2010

7) Killory BD, Nakaji P, Gonzales LF, Ponce FA, Wait SD, Spetzler RF. Prospective evaluation of surgical microscope-integrated intraoperative near-infrared indocyanine green angiography during cerebral arteriovenous malformation surgery. Neurosurgery 65: 456-462, 2009

8) Okawa M, Abe H, Hirata Y, et al.: [Visualization of the distal edge of carotid plaque using indocyanine green videoangiography during carotid endarterectomy and the pitfall involved]. No Shinkei Geka 39: 563-568, 2011 (Japanese)

9) Kodama Y, Ohnishi H, Taomoto K, et al.: Evaluation for perfusion areas of bypass flow by intraoperative near-infrared indocyanine green videoangiography in superficial temporal artery-middle cerebral artery bypass surgery. Surgery for Cerebral Stroke 37: 233-239, 2009 (Japanese)

10) Woitzik J, Horn P, Vajkoczy P, Schmiedek P: Intraoperative control of extracranial-intracranial bypass patency by near-infrared indocyanine green videoangiography. J Neurosurg 102: 692-698, 2005

11) Hunt WE, Hess RM: Surgical risk as related to time of intervention in the repair of intracranial aneurysms. J Neurosurg 28: 14-20, 1968 
12) Horie $N$, So G, Debata $A$, et al.: Intra-arterial indocyanine green angiography in the management of spinal arteriovenous fistulae: technical case reports. Spine 37: 264-267, 2012

13) van Swieten JC, Koudstaal PJ, Visser MC, Schouten HJ, van Gijn J: Interobserver agreement for the assessment of handicap in stroke patients. Stroke 19: 604-607, 1988

14) Schuette AJ, Cawley CM, Barrow DL: Indocyanine green videoangiography in the management of dural arteriovenous fistulae. Neurosurgery 67: 658-662; discussion 662, 2010

15) d'Avella E, Volpin F, Manara R, Scienza R, Della Puppa A: Indocyanine green videoangiography (ICGV)-guided surgery of parasagittal meningiomas occluding the superior sagittal sinus (SSS). Acta Neurochir (Wien) 155: 415-420, 2013

16) Ferroli P, Acerbi F, Albanese E, et al.: Application of intraoperative indocyanine green angiography for CNS tumors: results on the first 100 cases. Acta Neurochir Suppl 109: 251-257, 2011

17) Fisher CM, Kistler JP, Davis JM: Relation of cerebral vasospasm to subarachnoid hemorrhage visualized by computerized tomographic scanning. Neurosurgery 6: 1-9, 1980

18) Motoyama $Y$, Gurung $P$, Takeshima $Y$, Nakagawa I, Park YS, Nakase H: Indocyanine green (ICG) videoangiography-guided dissection of the sylvian fissure on the transsylvian approach: technical note. World Neurosurg 87: 45-47, 2016

Address reprint requests to: Hiroyuki Toi, MD, Department of Neurosurgery, Kawasaki Medical School, 577 Matsushima, Kurashiki, Okayama 701-0192, Japan.

email: ht11251974@yahoo.co.jp 